\title{
Probing mechanobiological role of filamin A in migration and invasion of human U87 glioblastoma cells using submicron soft pillars
}

\author{
Abdurazak Aman Ketebo ${ }^{1}$, Chanyong Park ${ }^{1}$, Jaewon Kim ${ }^{1}$, Myeongjun Jun ${ }^{1}$ and Sungsu Park ${ }^{1,2,3,4^{*}}$ (i)
}

\begin{abstract}
Filamin A (FLNa) belongs to an actin-binding protein family in binding and cross-linking actin filaments into a threedimensional structure. However, little attention has been given to its mechanobiological role in cancer cells. Here, we quantitatively investigated the role of FLNa by analyzing the following parameters in negative control (NC) and FLNaknockdown (KD) U87 glioma cells using submicron pillars (900 nm diameter and $2 \mu \mathrm{m}$ height): traction force (TF), rigidity sensing ability, cell aspect ratio, migration speed, and invasiveness. During the initial phase of cell adhesion $(<1 \mathrm{~h})$, FLNa-KD cells polarized more slowly than did NC cells, which can be explained by the loss of rigidity sensing in FLNa-KD cells. The higher motility of FLNa-KD cells relative to NC cells can be explained by the high TF exerted by FLNa-KD cells when compared to NC cells, while the higher invasiveness of FLNa-KD cells relative to NC cells can be explained by a greater number of filopodia in FLNa-KD cells than in NC cells. Our results suggest that FLNa plays important roles in suppressing motility and invasiveness of U87 cells.
\end{abstract}

Keywords: Filamin A, Traction force, Rigidity sensing, Cell adhesion, Motility, Filopodia

\section{Introduction}

Metastatic cells are highly motile and undergo cell morphology changes that facilitate penetration through the three-dimensional (3D) structures of the extracellular matrix (ECM). During invasion, metastatic cells form thin cellular protrusions called filopodia $[1,2]$ in the direction of movement to form focal adhesions (FAs) [3]. Filopodia play an important role in cell migration by sensing and forming initial contact with ECM. The primary function of FA is to transmit traction forces (TFs) through actin filaments and attach cells to the ECM [4]. In addition, metastatic cells must be able to attach and survive in different or secondary sites with ECM of variable stiffness $[5,6]$. However, the physical mechanisms by which metastatic cancer cells move through 3D structures of ECM

\footnotetext{
*Correspondence: nanopark@skku.edu

${ }^{4}$ School of Mechanical Engineering, Sungkyunkwan University (SKKU),

2066 Seobu-ro, 16419 Suwon, Korea

Full list of author information is available at the end of the article
}

and survive in ECM with variable stiffness are largely unknown. Understanding these mechanisms ultimately requires technology that can accurately quantify the TFs and rigidity sensing ability of cancer cells.

TF microscopy that uses micron or submicron pillars [7-9] made of the elastomeric polymer polydimethylsiloxane (PDMS) can be used to quantify TFs and rigidity sensing of cells (Fig. 1). Tangential tension by a cell deflects the pillar. The degree of deflection and the bending stiffness of the pillar are used to quantify TFs exerted by the cell [10]. Recent studies [11] showed that cells sense the rigidity of ECM by producing local contractions through actin-myosin interactions. These local contractions can be detected by observing the deflection of two neighboring pillars towards each other (Fig. 1A). TF microscopy showed that cancerous cells generated higher TFs than non-cancerous cells [12]. However, it has not been determined whether mutations in cancer cells that increase metastasis, motility and invasion can also increase TFs. 


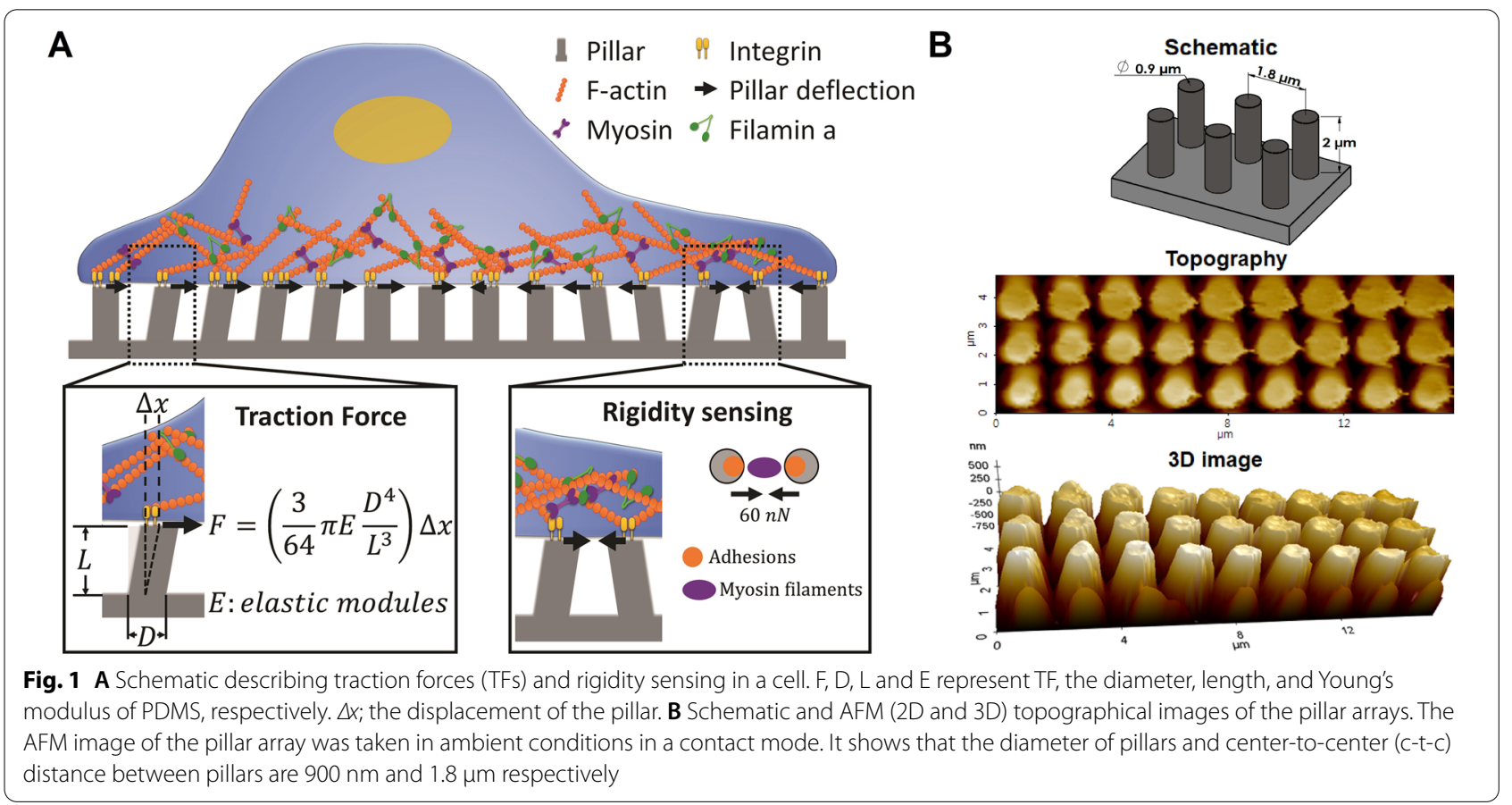

Filamin A (FLNa) is a V-shaped actin-binding protein that is abundantly expressed in human cells (Fig. 1A). It binds and cross-links cortical actin filaments into a 3D structure [13] and mediates the conversion of mechanical forces into biochemical signals [14]. It also links integrins to the actin filaments $[15,16]$. Thus, it integrates the cellular architecture and signaling that are essential for cell locomotion and development [17]. FLNa expression or suppression may facilitate or inhibit cancer invasion depending on the cell type, FLNa expression levels, and FLNa's interactions with other proteins. For example, the migration speed of FLNa-deficient melanoma and breast cancer cells were significantly lower than wild type cells [18]. In contrast, FLNa-knockdown (FLNa-KD) increased the frequency of multifocal tumors of glioma cell lines in immunodeficient mice, and it increased the migration speeds of other glioma cell lines (A172 and LN-229) in vitro [19]. The contradictory results from these studies show that the role of FLNa in cancer metastasis and invasion remains elusive [20].

Here, we evaluated the role of FLNa in cancer invasion by measuring TFs and rigidity sensing of negative control (NC) and FLNa-KD glioma (U87) cells. We used submicron pillars (diameter, $900 \mathrm{~nm}$; height, $2 \mu \mathrm{m}$; and center-to-center (c-t-c) distance between pillars, $1.8 \mu \mathrm{m}$; bending stiffness, $\mathrm{k}=24.2 \mathrm{nN} / \mu \mathrm{m}$ ) (Fig. $1 \mathrm{~B}$ ). The migratory and invasive behaviors of these cells were studied in conventional motility and invasion assays to demonstrate that mechanobiological results can be used as biomarkers for cancer metastasis.

\section{Experimental}

\subsection{Cell culture and transfection}

The Uppsala 87 malignant glioma (U87) cell line was purchased from American Type Culture Collection (ATCC, Manassas, VA). Cells were incubated in Gibco $1 \times$ minimum essential medium (Thermo Fisher Scientific) containing $10 \%$ fetal bovine serum (FBS) $\left(\right.$ Gibco $^{\circledR}$, Thermo Fisher Scientific) and 100 units $/ \mathrm{ml}$ penicillin (SigmaAldrich) under humid conditions at $37^{\circ} \mathrm{C}$ in $5 \% \mathrm{CO}_{2}$. Cells were transfected with either AccuTarget ${ }^{\mathrm{TM}}$ FLNa small interfering RNA (siRNA) oligonucleotide (target sequence: 5'-AAGATGGATTGCCAGGAGTG-3') or AccuTarget $^{\mathrm{TM}}$ negative control (NC) siRNA from Bioneer Co. (Daejeon, Korea) according to the manufacturer's instructions.

\subsection{Western blot analysis}

The total extract of NC and FLNa-KD cells was prepared using radioimmunoprecipitation assay buffer (LPS solution, Daejeon, Korea) and protease inhibitor cocktail (Roche). Anti-FLNa antibody from rabbit (Abcam) and anti-rabbit antibody conjugated with horseradish peroxidase were used as primary and secondary antibodies to visualize FLNa. Glyceraldehyde 3-phosphate dehydrogenase (GAPDH) was used as control. The image of the blot was captured using a chemiluminescent imaging system 
(Atto, Daejeon, Korea) and analyzed using Image (NIH, Bethesda, MD).

\subsection{Pillar fabrication}

Photolithography with reactive ion etching (RIE) was used to fabricate a mold of linearly arranged arrays of holes $(900 \mathrm{~nm}$, diameter; $2 \mu \mathrm{m}$, depth; and $1.8 \mu \mathrm{m}$, c-t-c distance between holes) over 5 -inch silicon wafers. PDMS Sylgrad $^{\circledR} 184$ silicon elastomeric base (Dow Corning Co.) [21] was mixed with its curing agent at either a 5:1 or 10:1 (w/w) ratio to obtain PDMS elastic modules of 4 and $2 \mathrm{MPa}$ [22] and the mixture was degassed for $15 \mathrm{~min}$. Next, the degassed mixture was spin-coated over the mold at $500 \mathrm{rpm}$ for $10 \mathrm{~s}$ and subsequently at $1,800 \mathrm{rpm}$ for $30 \mathrm{~s}$ before being degassed for $30 \mathrm{~min}$ to remove any trapped bubbles from the coated layer. The coated mold was cured at $80{ }^{\circ} \mathrm{C}$ for $4 \mathrm{~h}$. Next, the PDMS layer was peeled off from the mold. The bending stiffness $(k)$ of pillars made of PDMS at 5:1 and 10:1 mixing ratios of the elastomeric base and its curing agent was calculated to be 48.3 and $24.2 \mathrm{nN} / \mu \mathrm{m}$ using the following equation based on the Euler-Bernoulli beam theory: [23]

$$
k=\frac{3}{64} \pi E \frac{D^{4}}{L^{3}}
$$

where $D, L$, and $E$ represent the diameter, length, and Young's modulus of PDMS, respectively. The pillar array was probed by an atomic force microscope (AFM) using XE-7 (Park Systems, Suwon, Korea). Imaging under ambient conditions was conducted in a contact mode with PPP-CONTSCR cantilever (NANOSENSORS ${ }^{\mathrm{TM}}$, Neuchatel, Switzerland) with a nominal force constant of $0.2 \mathrm{~N} / \mathrm{m}$ and a nominal resonance frequency of $25 \mathrm{kHz}$.

\subsection{TF measurements}

To observe the deflection of pillars by cells, images of pillars with cells were obtained at 1 and $6 \mathrm{~h}$ in a live cell chamber (Live Cell Instrument, Seoul, Korea) at $37{ }^{\circ} \mathrm{C}$ with $5 \% \mathrm{CO}_{2}$ using a DeltaVision microscope (GE healthcare) equipped with a CoolSnap $\mathrm{HQ}^{2}$ camera (Photometrics, Tucson, AZ). PillarTracker version 1.1.3, which was obtained from the Mechanobiology Institute (MBI) in Singapore, was used to measure pillar deflection. PillarTracker applies a reconstruction algorithm to create a perfect grid for measuring pillar deflection. Pillars outside each cell were used as the reference corresponding to the zero-force position. The deflection value was multiplied by the bending stiffness of the pillar to determine the pillar's traction force (Fig. 1A).

\subsection{Rigidity sensing}

The rigidity sensing ability of a cell was determined by calculating the directionality parameter $(\gamma)$ during the initial phase of spreading $(<30 \mathrm{~min})$ at the leading edges (an area of $34.5 \mu \mathrm{m}^{2}$ ) of the boundary of the cell as a measure of local contractions [24]. $\gamma$ is calculated as the sum of the force vectors of pillars divided by the sum of their magnitudes. For this purpose, cell images taken within 30 min of cell substrate contact were used for $\gamma$ calculation. Average $\gamma$ values of each type of cells were obtained by calculating fifty frames of six different cells.

\subsection{Fluorescent imaging of F-actin}

Cells were incubated on fibronectin-coated pillars in an incubator containing $5 \% \mathrm{CO}_{2}$ at $37^{\circ} \mathrm{C}$ for 1 and $6 \mathrm{~h}$. The cells were washed with phosphate-buffered saline (PBS) (pH 7.4) and fixed with 4\% paraformaldehyde in PBS at room temperature (RT) for $10 \mathrm{~min}$. Cells were permeabilized with $0.5 \%$ triton X-100 in PBS at RT for 5 min. F-actin was first stained with rhodamine phalloidin (RP) (Sigma-Aldrich) $(1: 1,000)$ in PBS at RT for $30 \mathrm{~min}$, and the nucleus was subsequently stained with $0.2 \mathrm{mg} / \mathrm{mL}$ of 4,6-diamidino-2-phenylindole (DAPI) (Sigma-Aldrich) in PBS at RT for 15 min.

\subsection{Measurement of cell aspect ratio on pillars}

Fluorescent images of cells stained with RP and DAPI were obtained. Cell shape was represented as an equivalent elliptical shape using ImageJ. The aspect ratio, which was used as a measure of cell polarity, was determined by dividing the major axis of the ellipse by the minor axis.

\subsection{Immunostaining of PXN and fascin}

For FA analysis, cells that were incubated on fibronectincoated glass slides in an incubator containing $5 \% \mathrm{CO}_{2}$ at $37{ }^{\circ} \mathrm{C}$ for 1 and $6 \mathrm{~h}$ were washed, fixed, and permeabilized as described above. The cells were immunostained with rabbit anti-PXN antibody (Abcam) for $1 \mathrm{~h}$. Next, they were incubated with anti-rabbit antibody conjugated with Alexa Fluor ${ }^{\circledR} 488$ (Thermo Fisher Scientific) for $45 \mathrm{~min}$. Finally, fluorescent images were taken using a DeltaVision microscope.

To image filopodia, cells that were incubated on fibronectin-coated pillars in an incubator containing 5\% $\mathrm{CO}_{2}$ at $37{ }^{\circ} \mathrm{C}$ were washed, fixed, and permeabilized as described above. They were immunostained with rabbit anti-fascin primary antibody (Abcam) for $1 \mathrm{~h}$. Next, they were incubated with anti-rabbit antibodies conjugated with Alexa Fluor ${ }^{\circledR} 488$ for 45 min. Finally, fluorescent images were taken using a DeltaVision microscope (GE Healthcare).

\subsection{Cell motility experiment and invasion assay}

Pillars were coated with fibronectin for $1 \mathrm{~h}$ before seeding cells. Cells were seeded at a density of 3000 cells per sample. They were tracked every $5 \mathrm{~min}$ for $6 \mathrm{~h}$ using a 
$\mathrm{JuLi}^{\mathrm{TM}} \mathrm{Br}$ live cell movie analyzer (NanoEntek, Inc., Seoul, Korea). The migration speed of NC and FLNa-KD cells $(n=10)$ were calculated after tracking the movement of the cell using ImageJ. An invasion assay was performed using modified Boyden Chambers BioCoat ${ }^{\mathrm{TM}}$ Matrigel $^{\circledR}$ Invasion Chamber (Corning) comprised of Matrigel membrane filter inserts with $8 \mu \mathrm{m}$ pores in a 24-well tissue culture plate [25]. A total of $2 \times 10^{4}$ cells were mixed with minimum essential medium (MEM) with $20 \%$ FBS and seeded onto the top of the chamber, and the bottom chamber was filled with MEM containing $20 \%$ FBS as a chemoattractant. The cells were incubated at $5 \% \mathrm{CO}_{2}$ and $37{ }^{\circ} \mathrm{C}$ for $24 \mathrm{~h}$. Non-invading cells were removed from the upper membrane using a cotton swab, and invasive cells were fixed with $4 \%$ paraformaldehyde and stained with toluidine blue (Sigma-Aldrich). Stained cells were counted randomly from three locations on the membrane. The experiment was repeated at least three times.

\subsection{Statistical analysis and error correction}

Throughout this study, all data were represented by the mean \pm standard error of the mean of the samples. The student's t-test was used to compare NC and FLNa-KD samples. A P-value of less than 0.05 was considered significant $\left({ }^{*} \mathrm{P}<0.05\right.$, ** $\mathrm{P}<0.01$, *** $\left.\mathrm{P}<0.001\right)$. In the $\mathrm{TF}$ experiments, the deflection errors of the pillars were reduced by subtracting the mean deflection of the empty pillars outside the cell boundary.

\section{Results and discussion}

\subsection{Delayed cell spreading due to impairment in rigidity sensing}

During initial cell adhesion, the leading edges of cells undergo periodic protrusion-retraction cycles to test ECM rigidity [26]. Cells control their spreading based on ECM rigidity [27]. To investigate the role of FLNa on cell spreading, the shape of NC and FLNa-KD cells incubated on fibronectin-coated pillars (Fig. 1B) for 1 and $6 \mathrm{~h}$ was observed with actin staining and analyzed by measuring their aspect ratio. Before incubation, FLNa knockdown was verified in cells using western blotting (Fig. 2A). At $1 \mathrm{~h}$, FLNa-KD cells were relatively round and less polarized than NC cells (Fig. 2B), which is supported by the fact that the cell aspect ratio of the FLNa-KD cells was significantly lower than that of the $\mathrm{NC}$ cells at $1 \mathrm{~h}(\mathrm{P}<0.001)$. At $6 \mathrm{~h}, \mathrm{FLNa}-\mathrm{KD}$ cells were elongated to a similar extent as NC cells (Fig. 2B), which is supported by the fact that there was no significant difference in the aspect ratio between NC and FLNa-KD cells (Fig. 2C). These results suggest that FLNa-KD can delay cell spreading when cells contact ECM for a limited period (about $1 \mathrm{~h}$ ) but the delayed effect of FLNa-KD in cell polarization diminished at $6 \mathrm{~h}$.

Cells sense the rigidity of the substrate by undergoing local contractions. It occurs during the initial phase of cell spreading ( $<30 \mathrm{~min})$. Local contractions by cells occur through contractile units (CUs) consisting of cytoskeletal protein complexes, including tropomyosin, myosin II, integrin, and $\alpha$-actinin (Fig. 1A) [28]. To understand why FLNa-KD cells delayed spreading on pillars (Fig. 2B), we investigated the effect of FLNa-KD on rigidity sensing by observing the deflection of pillars at the leading edges (an area of $34.5 \mu^{2}$ ) of the boundary of NC and FLNa-KD cells at $<30 \mathrm{~min}, 60 \mathrm{~min}$, and $360 \mathrm{~min}$ [29]. For $<30 \mathrm{~min}$, locally contracted pillars were observed on the leading edges of the NC cells but not on the leading edges of FLNa-KD cells (Fig. 2D). At 60 and $360 \mathrm{~min}$, all pillars were centripetally deflected in both NC and FLNa-KD cells. The deflected direction of pillars was further analyzed to determine the directionality parameter $(\gamma) . \gamma$ is 1 when the force vectors of two neighboring pillars are parallel. $\gamma$ is 0 when the force vectors of the pillars are locally contracted toward each other. At $<30 \mathrm{~min}, \gamma$ of the NC cells was $0.4 \pm 0.14$ (mean \pm standard deviation (SD)), indicating that they sensed the rigidity of the pillars $(k=24.2 \mathrm{nN} / \mu \mathrm{m})$ (Fig. 2E). However, $\gamma$ of the FLNa-KD cells was $0.9 \pm 0.03$ (mean $\pm \mathrm{SD}$ ) (Fig. $2 \mathrm{E}$ ), indicating that they could not sense the rigidity of the pillars [29]. At 60 and $360 \mathrm{~min}$, the $\gamma$ values of both $\mathrm{NC}$ and FLNa-KD cells were about 0.9 . This result shows that the local contraction was observed only during the initial phase of spreading for U87 cells with intact FLNa. Similarly, the local contraction by $\mathrm{NC}$ triple negative breast cancer (MDA-MB-231) cell was observed on pillars $(k=24.2 \mathrm{nN} / \mu \mathrm{m})$ for $<30 \mathrm{~min}$ (Additional file 1: Figure $\mathrm{S} 1 \mathrm{~A}, \mathrm{~B})$, demonstrating that the local contraction of the pillar is not dependent on the bending stiffness of pillars. $\mathrm{CU}$ proteins like FLNa and tropomyosin are required to exert local contractions in cells [30]. Most cancer cells including glioma cells are known to have decreased tropomyosin expression. Together with the previous report, our results imply that the NC U87 cells do not completely lose their rigidity sensing. Cells can grow on substrate with low rigidity even when their rigidity sensing is completely impaired [31]. The loss of rigidity sensing is advantageous for metastatic cell survival in different or secondary sites with variable stiffness of the ECM. In particular, FLNa plays important roles in stabilizing early adhesion sites through its direct binding to integrin. Thus, FLNa-KD might have delayed the recruitment of proteins to adhesion sites, including $\alpha$-actinin, actin filaments, and tropomyosin [32]. CUs cannot form without these proteins. This may explain why locally contracted pillars were not observed in the FLNa-KD cells. Taken 
A

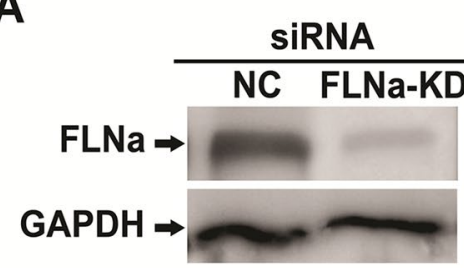

C

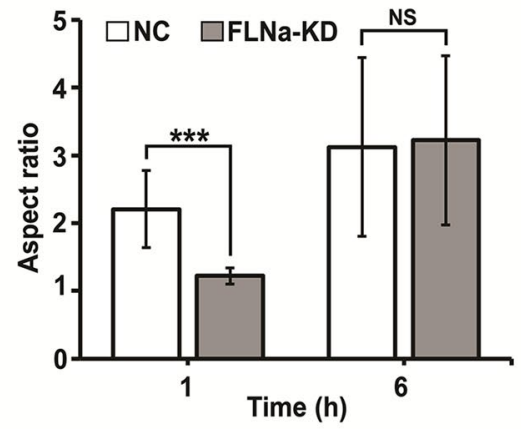

D

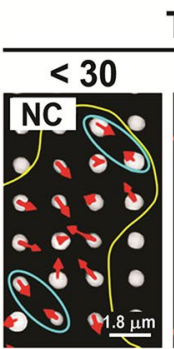

Time ( $\min )$

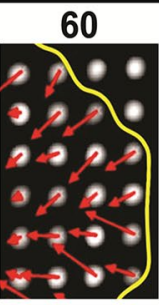

360

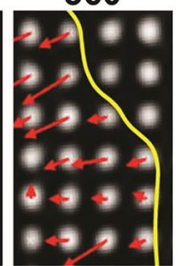

FLNa-KD?

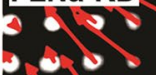

300

30,0

- 01

) $J_{2.8 \mathrm{nN}}$

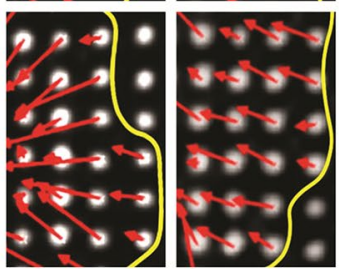

280

36
B

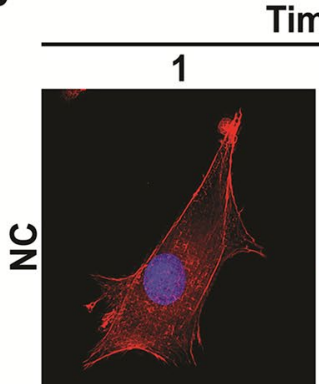

Time (h)

MM (kD)
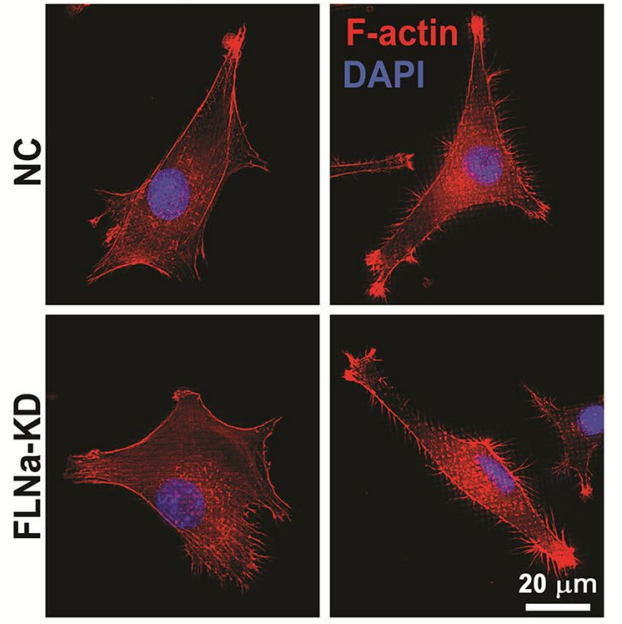

E

$\square$ NC $\square$ FLNa-KD

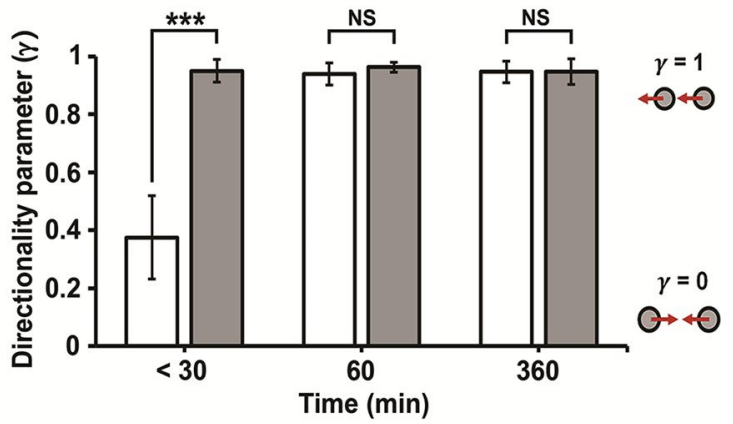

Fig. 2 Cell spreading and rigidity sensing ability of NC and FLNa-KD cells on fibronectin-coated pillars (diameter, $900 \mathrm{~nm}$; height, 2 m; and pillar center-to-center distance, $1.8 \mu \mathrm{m}$; bending stiffness $k=24.2 \mathrm{nN} / \mu \mathrm{m}$ ). A Western blot of FLNa. Control: GAPDH. B Fluorescence images of F-actin (RP: red) and the nuclei (DAPI: blue) of cells on fibronectin-coated pillars at 1 and 6 h. C Aspect ratio of cells. ( $n=30$ cells). D Deflection of pillars in NC and FLNa-KD cells near the edge (approximately $34.5{\mu m^{2}}^{2}$ ) of cells at $<30,60$, and 360 min incubations. Local contractions in the NC cells are marked with cyan circles. The red arrows indicate pillar deflection. E Directionality parameters ( $\gamma$ ) of the NC and FLNa-KD cells at $<30,60$, and 360 min incubations. $y$ is calculated as the sum of the force vectors of pillars divided by the sum of their magnitudes. $y$ is 1 when the force vectors of two neighboring pillars are parallel. $y$ is 0 when the force vectors of the pillars are locally contracted toward each other. Cell number $n=6$. Error bar represents the mean \pm standard deviation (SD). ${ }^{* *} \mathrm{P}<0.001$. Student's t-test. NS Not significant

together, these data suggest that the delay in spreading in the FLNa-KD cells during the initial phase of cell spreading (Fig. 2B, C) could be due to the loss of rigidity sensing (Fig. 2D, E).

\subsection{High motility and traction force in FLNa-KD cells}

Metastatic properties of cancer cells like high motility can be studied by quantifying individual cell motilities.
Using fibronectin-coated pillars the motility of individual cell was monitored for $6 \mathrm{~h}$. The FLNa-KD cells migrated faster than the NC cells at 1 and $6 \mathrm{~h}$ (Fig. 3A), indicating that FLNa-KD increased cell motility.

Cell mechanics and migration are known to be directly related [33-35]. TFs in FLNa-KD and NC cells were compared to quantitatively describe how FLNa-KD increased cell migration speed. TFs exerted by the FLNa-KD and 
A

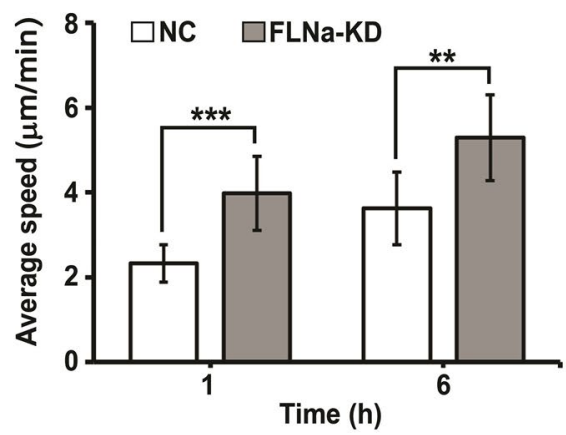

C

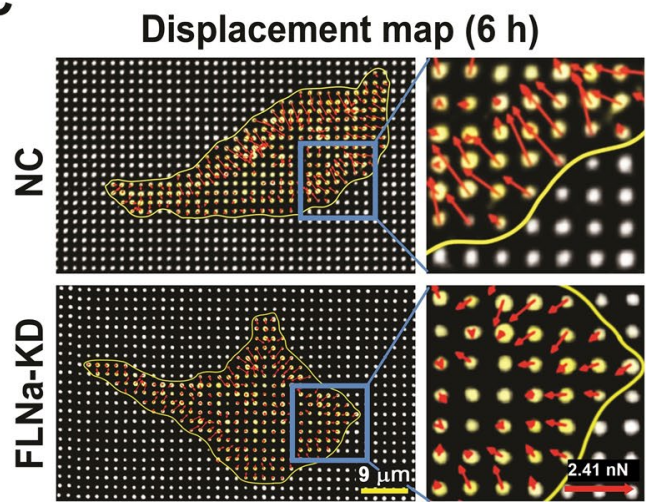

B

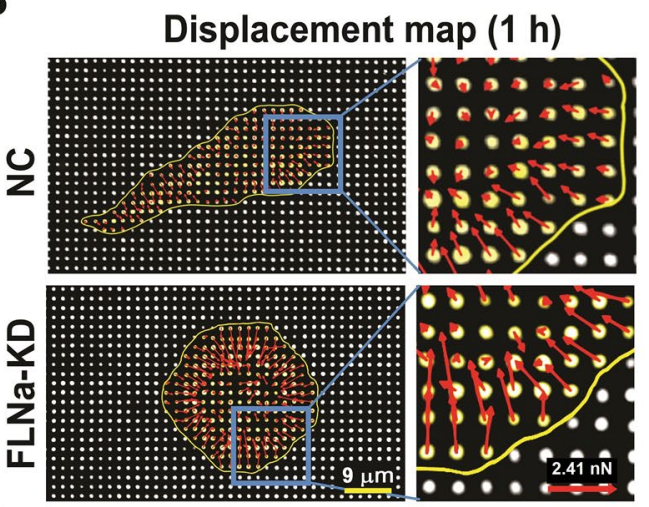

$\mathbf{D}$

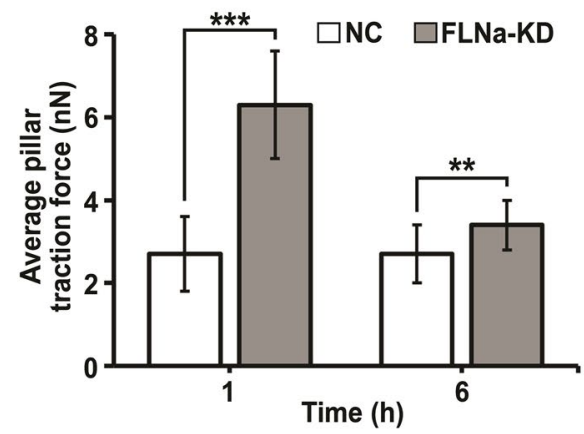

Fig. 3 Motility and TFs of NC and FLNa-KD cells on fibronectin-coated pillars (A) Average speed of cells $(n=10$ cells) at 1 and $6 \mathrm{~h}$ of incubation on the pillars. The representative deflection maps $(\mathbf{B}, \mathbf{C})$ of the $N C$ and FLNa-KD cells on pillars at $1 \mathrm{~h}(\mathbf{B})$ and $6 \mathrm{~h}(\mathbf{C})$. The yellow line is the approximate boundary of the cells. The red arrow indicates the deflection magnitude and direction of each pillar. (D) Average TFs of the cells at 1 and $6 \mathrm{~h}$. Number of samples, $\left(n=40\right.$ cells). Error bar represents the mean $\pm S D ;{ }^{* *} P<0.01$, ${ }^{* * *} P<0.001$; Student's t-test

$\mathrm{NC}$ cells were calculated using the deflection data of each pillar with the bending stiffness $(k=24.2 \mathrm{nN} / \mu \mathrm{m})$. At both 1 and $6 \mathrm{~h}$, the TFs of the FLNa-KD cells were significantly higher than the NC cells (Fig. 3D) (Additional file 2: Movie. S1 and Additional file 3: Movie. S2). A similar result was also observed in MDA-MD-231 cells (Additional file 1: Figure S2). Since FLNa competes with talin to bind with integrin $[36,37]$, talin binding to the integrin $\beta$ tail increases when FLNa is impaired and thus enhances integrin activation and cell motility through FA turnover. These results suggest that FLNA-KD cells increase their motility by exerting high TFs.

\subsection{Effect of FLNa-KD on cell FA}

FAs comprise several kinds of proteins, including filamin (FLNa) and paxillin (PXN), which perform different functions. For example, integrins are signaling proteins that link the ECM with the cell cytoskeleton [38]. PXN is an FA protein that controls cell spreading and motility [39]. FLNa is known to mediate the phosphorylation PXN in androgen-stimulated cells [40].
In addition, PXN is a well-known FA marker [41]. To determine if FAs of cells were affected by FLNa-KD, FA size and number were analyzed in NC and FLNa$\mathrm{KD}$ cells at 1 and $6 \mathrm{~h}$ after cell incubation on the glass surface by immunostaining PXN [42], an FA protein. As shown in Fig. 4A, the FAs of NC appears brighter and greater in number compared to NC cells at 1 and $6 \mathrm{~h}$. Both the FA number and size of the FLNa-KD cells were lower than NC cells at 1 and $6 \mathrm{~h}$ (Fig. 4B, C), suggesting that FLNa-KD inhibited FA maturation [43]. Previously, it was reported that FA size was negatively related to TFs [44]. Low levels of FLNa expression can increase cancer cell migration by regulating FA assembly and disassembly (FA turnover). The small FA size could be due to the inability of microtubules to extend to the periphery of FLNa-KD cells [45, 46]. Additionally, it was reported that small FAs apply strong TFs to drive cell migration while large FAs anchor the cell to the substrate and produce weak TFs. The results suggest that reduced FA size in the FLNa-KD cells reflects high motility. 

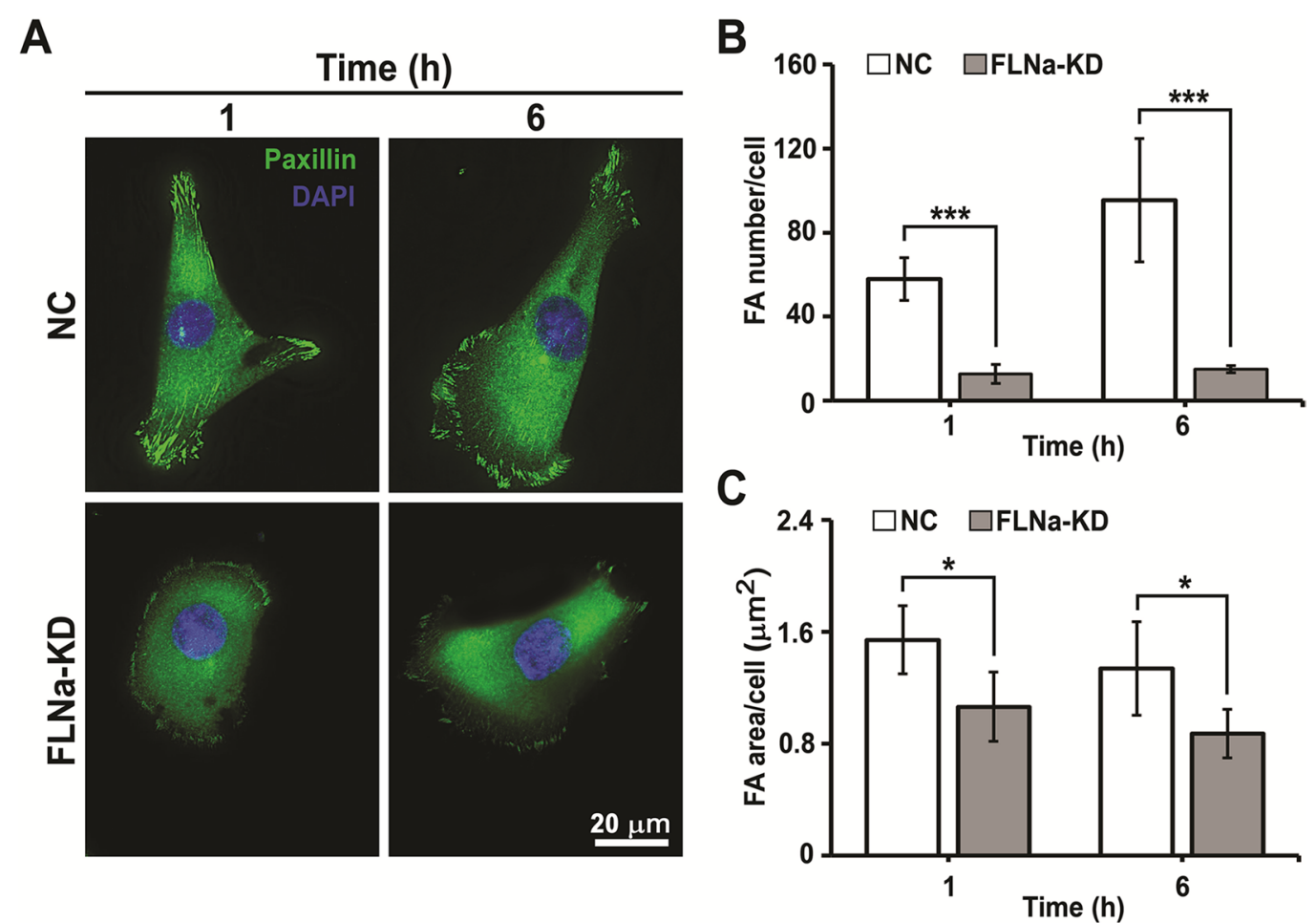

Fig. 4 FA number and size of NC and FLNa-KD cells on glass slides coated with fibronectin at 1 and $6 \mathrm{~h}$. $\mathbf{A}$ Immunostaining of paxillin (PXN, green) in cells. FA number $(\mathbf{B})$ and size $(\mathbf{C})$. Error bar represents the mean $\pm S D$. $\left(n=6\right.$ cells). ${ }^{*} P<0.05$; ${ }^{* * *} P<0.001$; Student's t-test

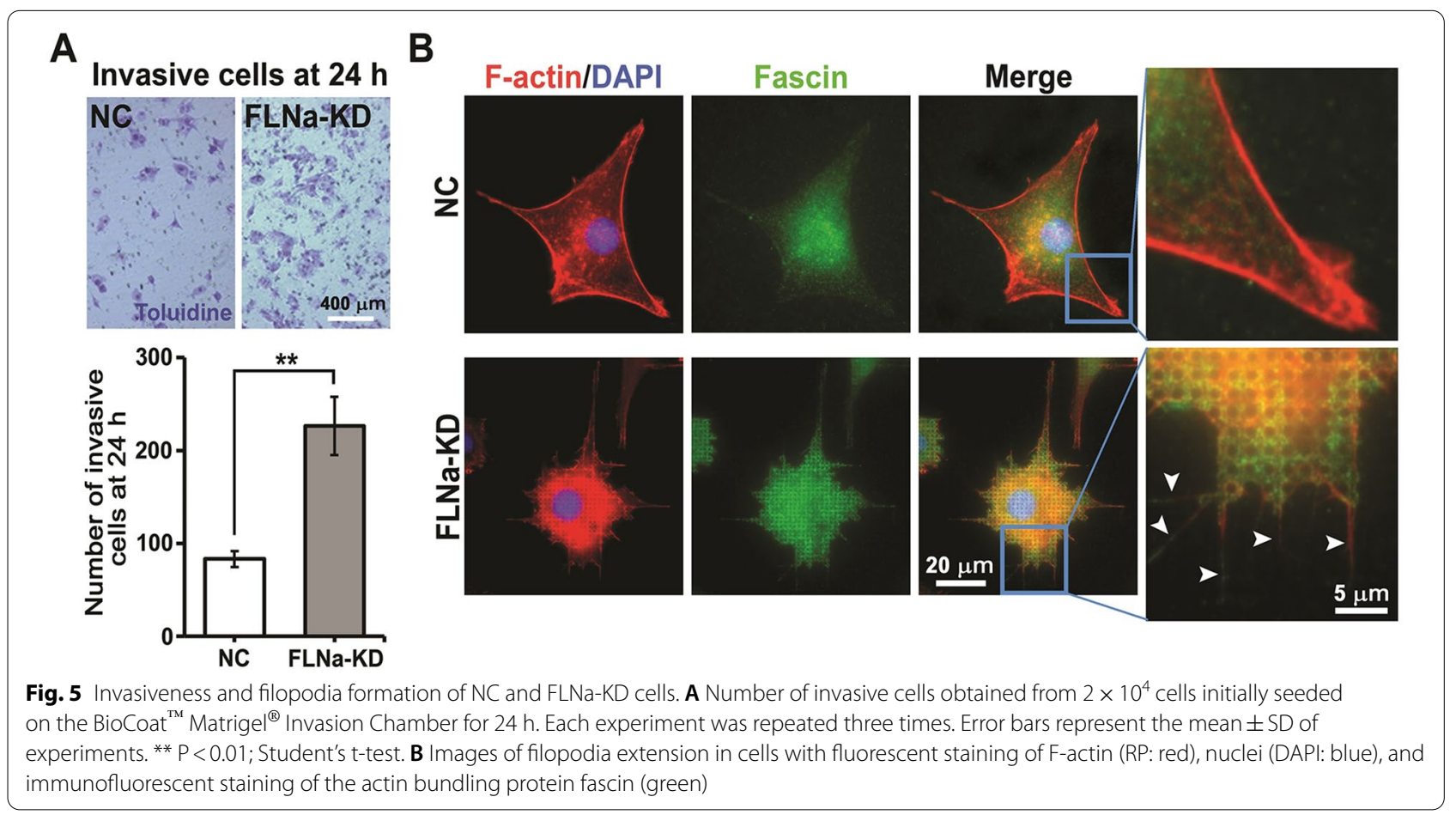




\subsection{Effect of FLNa-KD on invasion}

The effect of FLNa on cell invasion was analyzed using the BioCoat ${ }^{\mathrm{TM}}$ Matrigel $^{\circledR}$ Invasion Chamber [47]. Figure $5 \mathrm{~A}$ shows that FLNa-KD cells are significantly more invasive than NC cells. During invasion, metastatic cells produce thin cellular protrusions called filopodia in the direction of movement to form FAs. Filopodia extensions have strong parallel actin microfilaments that are bundled together by an actin bundling protein called fascin. The immunostaining images of fascin show that FLNa-KD cells displayed a higher number of filopodia extensions than did NC cells on pillars at $1 \mathrm{~h}$ (Fig. 5B indicated by white arrows). The difference was not obvious on pillars at $6 \mathrm{~h}$ (data not shown). Filopodia are related to cancer invasion. For instance, filopodia inhibition in malignant glioma cells produced a decrease in invasiveness [48]. Taken together, this study showed the migratory ability and invasiveness of FLNa-KD cells using mechanobiological measurements.

\section{Conclusions}

Previous studies showed that FLNa expression or suppression may facilitate or inhibit cancer invasion depending on the cell type, FLNa expression levels, and FLNa interactions with other proteins. Here, TF microscopy using submicron pillars showed that FLNa-KD impairs rigidity sensing and delays cell spreading during the initial phase of cell spreading in U87, while the conventional methods of immunostaining and motility assays showed that the KD facilitated motility and invasion. Our current findings demonstrate that mechanobiological parameters like TF and rigidity sensing can be used to explain the physical mechanisms of highly migratory and invasive properties of metastatic cancer cells.

\section{Abbreviations}

c-t-c: Center-to-center; CU: Contractile unit; DAPI: 4,6-diamidino-2-phenylindole; ECM: Extracellular matrix; FA: Focal adhesion; FBS: Fetal bovine serum; FLNa: Filamin a; GAPDH: Glyceraldehyde 3-phosphate dehydrogenase; KD: Knockdown; MEM: Minimum essential medium; NC: Negative control; PXN: Paxillin; PBS: Phosphate-buffered saline; PDMS: Polydimethylsiloxane; RP: Rhodamine phalloidin; RT: Room temperature; siRNA: Small interfering RNA.

\section{Supplementary Information}

The online version contains supplementary material available at https://doi. org/10.1186/s40580-021-00267-6.

Additional file 1: Figure S1. Rigidity sensing ability of NC and FLNa-KD MDA-MB-231 cells. Figure S2. Average TF of of NC and FLNa-KD MDAMB-231 cells after 1 h-incubation on pillars.

Additional file 2. NC U87 cells spreading on pillars with the bending stiffness $(k=24.2 \mathrm{nN} / \mu \mathrm{m})$ for $3 \mathrm{~min}$ after the cell attached to the top surface of the pillars.

Additional file 3. FLNa-KD cells spreading on pillars with the bending stiffness $(k=24.2 \mathrm{nN} / \mu \mathrm{m})$ for 3 min after the cell attached to the top surface of the pillars.

\section{Acknowledgements}

We are grateful for the technical help from X. Xu and F. Margadant at Mechanobiology Institute (MBI), National University of Singapore (NUS). We thank Professor M.P. Sheetz at MBI for the valuable discussion regarding actomyosin's role in cancer.

\section{Authors' contributions}

AAK performed most of the experiments. CP conducted the AFM experiment, JK and MJ analyzed data regarding traction force. AAK and SP are major contributors in writing the manuscript. All authors read and approved the final manuscript.

\section{Funding}

This work was equally supported by the National Research Foundation (NRF) grants (2018M3A6B2057299, 2019K2A9A2A12000343) through the Ministry of Science and ICT (MSIT) in Korea and Technology Innovation Program (20008413, Development of disease models based on 3D microenvironmental platform mimicking multiple organs and evaluation of drug efficacy) through the Ministry of Trade, Industry \& Energy (MOTIE) in Korea.

\section{Availability of data and materials}

All data generated or analyzed during this study are included in this published article and its supplementary information files.

\section{Declarations}

\section{Competing interests}

The authors declare that they have no competing interests.

\section{Author details}

${ }^{1}$ Department of Mechanical Engineering, Sungkyunkwan University (SKKU), 16419 Suwon, Korea. ${ }^{2}$ Department of Biomedical Engineering, Sungkyunkwan University (SKKU), 16419 Suwon, Korea. ${ }^{3}$ Institute of Quantum Biophysics (IQB), Sungkyunkwan University (SKKU), 16419 Suwon, Korea. ${ }^{4}$ School of Mechanical Engineering, Sungkyunkwan University (SKKU), 2066 Seobu-ro, 16419 Suwon, Korea.

Received: 22 March 2021 Accepted: 21 May 2021

Published online: 02 July 2021

References

1. J.C. Adams, Roles of fascin in cell adhesion and motility. Curr. Opin. Cell Biol. 16, 590 (2004). https://doi.org/10.1016/j.ceb.2004.07.009

2. J.R. Bartles, Parallel actin bundles and their multiple actin-bundling proteins. Curr. Opin. Cell Biol. 12, 72 (2000). https://doi.org/10.1016/S09550674(99)00059-9

3. Y. Xu, T.A. Bismar, J. Su, B. Xu, G. Kristiansen, Z. Varga, L. Teng, D.E. Ingber, A. Mammoto, R. Kumar, M.A. Alaoui-Jamali, Filamin A regulates focal adhesion disassembly and suppresses breast cancer cell migration and invasion. J. Exp. Med 207, 2421 (2010). https://doi.org/10.1084/jem.20100 433

4. S.K. Sastry, K. Burridge, Focal Adhesions: A Nexus for Intracellular Signaling and Cytoskeletal Dynamics. Exp. Cell Res. 261, 25 (2000). https://doi.org/ 10.1006/excr.2000.5043

5. P.S. Steeg, Tumor metastasis: mechanistic insights and clinical challenges. Nat. Med. 12, 895 (2006). https://doi.org/10.1038/nm1469

6. I.J. Fidler, The pathogenesis of cancer metastasis: the "seed and soil" hypothesis revisited. Nat. Rev. Cancer 3, 453 (2003). https://doi.org/10. 1038/nrc1098

7. T.H. Shin, A.A. Ketebo, S. Lee, S.H. Kang, S. Basith, B. Manavalan, D.H. Kwon, S. Park, G. Lee, Decrease in membrane fluidity and traction force induced by silica-coated magnetic nanoparticles. J. Nanobiotechnol 19, 1 (2021). https://doi.org/10.1186/s12951-020-00765-5

8. Y. Cui, F.M. Hameed, B. Yang, K. Lee, C.Q. Pan, S. Park, M. Sheetz, Cyclic stretching of soft substrates induces spreading and growth. Nat. Commun. 6, 1 (2015). https://doi.org/10.1038/ncomms7333 
9. Y.M. Park, J. Ahn, Y.S. Choi, J.-M. Jeong, S.J. Lee, J.J. Lee, B.G. Choi, K.G. Lee, Flexible nanopillar-based immunoelectrochemical biosensor for noninvasive detection of amyloid beta. Nano Convergence 7, 1 (2020). https://doi. org/10.1186/s40580-020-00239-2

10. J.L. Tan, J. Tien, D.M. Pirone, D.S. Gray, K. Bhadriraju, C.S. Chen, Cells lying on a bed of microneedles: an approach to isolate mechanical force. Proc. Natl. Acad. Sci. U. S. A. 100, 1484 (2003). https://doi.org/10.1073/pnas.0235407100

11. M. Saxena, S. Liu, B. Yang, C. Hajal, R. Changede, J. Hu, H. Wolfenson, J. Hone, M.P. Sheetz, EGFR and HER2 activate rigidity sensing only on rigid matrices. Nat. Rev. Mater. 16, 775 (2017). https://doi.org/10.1038/nmat4893

12. C.T. Mierke, B. Frey, M. Fellner, M. Herrmann, B. Fabry, Integrin A5B1 facilitates cancer cell invasion through enhanced contractile forces. J. Cell Sci. 124, 369 (2011). https://doi.org/10.1242/jcs.071985

13. G.M. Popowicz, M. Schleicher, A.A. Noegel, T.A. Holak, Filamins: promiscuous organizers of the cytoskeleton. Trends Biochem. Sci. 31, 411 (2006). https:// doi.org/10.1016/j.tibs.2006.05.006

14. A.J. Kosmalska, L. Casares, A. Elosegui-Artola, J.J. Thottacherry, R. MorenoVicente, V. González-Tarragó, M.Á. Del Pozo, S. Mayor, M. Arroyo, D. Navajas, X. Trepat, N.C. Gauthier, P. Roca-Cusachs, Physical principles of membrane remodelling during cell mechanoadaptation. Nat. Commun. (2015). https:// doi.org/10.1038/ncomms8292

15. HToyoda, KIna, H Kitamura, TTsuda, and T Shimada, Organization of the Lamina Propria Mucosae of Rat Intestinal Mucosa, with Special Reference to the Subepithelial Connective Tissue. Acta Anat. (Basel). 158, 172 (1997). https://doi.org/10.1159/000147928

16. E. Puklin-Faucher, M.P. Sheetz, The Mechanical integrin cycle. J. Cell Sci. 122, 179 (2009). https://doi.org/10.1242/jcs.042127

17. T.P. Stossel, J. Condeelis, L. Cooley, J.H. Hartwig, A. Noegel, M. Schleicher, S.S. Shapiro, Filamins as integrators of cell mechanics and signalling. Nat. Rev. Mol. Cell Bio. 2, 138 (2001). https://doi.org/10.1038/35052082

18. X. Jiang, J. Yue, H. Lu, N. Campbell, Q. Yang, S. Lan, B.G. Haffty, C. Yuan, Z Shen, Inhibition of filamin-A reduces cancer metastatic potential. Int. J. Biol. Sci. 9, 67 (2012). https://doi.org/10.7150/ijbs.5577

19. J. Yang, J. Fan, Y. Li, F. Li, P. Chen, Y. Fan, X. Xia, S.T. Wong, Genome-Wide RNAi screening identifies genes inhibiting the migration of glioblastoma cells. PLoS ONE (2013). https://doi.org/10.1371/journal.pone.0061915

20. J. Yue, S. Huhn, Z. Shen, Complex roles of filamin-A mediated cytoskeleton network in cancer progression. Cell Biosci. 3, 7 (2013). https://doi.org/10. 1186/2045-3701-3-7

21. S.G. Mun, H.W. Choi, J.M. Lee, J.H. Lim, J.H. Ha, M.-J. Kang, E.-J. Kim, L. Kang, B.G. Chung, RGO nanomaterial-mediated cancer targeting and photothermal therapy in a microfluidic co-culture platform. Nano Convergence 7, 1 (2020). https://doi.org/10.1186/s40580-020-0220-3

22. Z. Wang, A.A. Volinsky, N.D. Gallant, Crosslinking effect on polydimethylsiloxane elastic modulus measured by custom-built compression instrument. J. Appl. Polym. Sci. 131, 1 (2014). https://doi.org/10.1002/app.41050

23. D.W. Zhou, T.T. Lee, S. Weng, J. Fu, A.J. García, Effects of substrate stiffness and actomyosin contractility on coupling between force transmission and vinculin-paxillin recruitment at single focal adhesions. Mol. Biol. Cell 28, 1901 (2017). https://doi.org/10.1091/mbc.E17-02-0116

24. A.A. Ketebo, T.H. Shin, M. Jun, G. Lee, S. Park, Effect of silica-coated magnetic nanoparticles on rigidity sensing of human embryonic kidney cells. J. Nanobiotechnol 18, 170 (2020). https://doi.org/10.1186/s12951-020-00730-2

25. A Valster, NL Tran, M Nakada, ME Berens, AY Chan, M Symons, and M Valster, A., Tran, N. L., Nakada, M., Berens, M. E., Chan, A. Y., Symons, Cell Migration and Invasion Assays. Methods. 37, 208 (2005). https://doi.org/10.1007/9781-61779-80-5_27

26. G. Meacci, H. Wolfenson, S. Liu, M.R. Stachowiak, T. Iskratsch, A. Mathur, S. Ghassemi, N. Gauthier, E. Tabdanov, J. Lohner, A. Gondarenko, A.C. Chander, P. RocaCusachs, B. O'Shaughnessy, J. Hone, M.P. Sheetz, a-actinin links extracellular matrix rigidity-sensing contractile units with periodic cell-edge retractions. Mol. Biol. Cell 27, 3471 (2016). https://doi.org/10.1091/mbc.E16-02-0107

27. A.D. Bershadsky, M.M. Kozlov, Crawling cell locomotion revisited. Proc. Natl. Acad. Sci. U. S. A. 108, 20275 (2011). https://doi.org/10.1073/pnas.1116814108

28. M. Saxena, R. Changede, J. Hone, H. Wolfenson, M.P. Sheetz, Force-induced calpain cleavage of talin is critical for growth, adhesion development, and rigidity sensing. Nano Lett. 17, 7242 (2017). https://doi.org/10.2147/NDT. S152247

29. S. Ghassemi, G. Meacci, S. Liu, A.A. Gondarenko, A. Mathur, P. Roca-Cusachs, M.P. Sheetz, J. Hone, Cells test substrate rigidity by local contractions on submicrometer pillars. Proc. Natl. Acad. Sci. U. S. A. 109, 5328 (2012). https:// doi.org/10.1073/pnas.1119886109

30. B. Yang, H. Wolfenson, V.Y. Chung, N. Nakazawa, S. Liu, J. Hu, R.Y.-J.J. Huang, M.P. Sheetz, Stopping transformed cancer cell growth by rigidity sensing. Nat. Rev. Mater. 19, 239 (2019). https://doi.org/10.1038/s41563-019-0507-0

31. A.W. Hamburger, S.E. Salmon, Primary bioassay of human tumor stem cells. Science 197, 461 (1977). https://doi.org/10.1126/science.560061

32. H. Wolfenson, G. Meacci, S. Liu, M.R. Stachowiak, T. Iskratsch, S. Ghassemi, P. Roca-Cusachs, B. O'Shaughnessy, J. Hone, M.P. Sheetz, Tropomyosin controls sarcomere-like contractions for rigidity sensing and suppressing growth on soft matrices. Nat. Cell Biol. 18, 33 (2016). https://doi.org/10.1038/ncb3277

33. L. Blanchoin, R. Boujemaa-Paterski, C. Sykes, J. Plastino, Actin dynamics, architecture, and mechanics in cell motility. Physiol. Rev. 94, 235 (2014). https:// doi.org/10.1152/physrev.00018.2013

34. F. Lautenschläger, S. Paschke, S. Schinkinger, A. Bruel, M. Beil, J. Guck, The regulatory role of cell mechanics for migration of differentiating myeloid cells. Proc. Natl. Acad. Sci. U. S. A. 106, 1569 (2009). https://doi.org/10.1073/ pnas.0811261106

35. B. Xuan, D. Ghosh, E.M. Cheney, E.M. Clifton, M.R. Dawson, Dysregulation in actin cytoskeletal organization drives increased stiffness and migratory persistence in polyploidal giant cancer cells. Sci. Rep. 8, 1 (2018). https://doi. org/10.1038/s41598-018-29817-5

36. T. Kiema, Y. Lad, P. Jiang, C.L. Oxley, M. Baldassarre, K.L. Wegener, I.D. Campbell, J. Ylänne, D.A. Calderwood, The molecular basis of filamin binding to integrins and competition with Talin. Mol. Cell 21, 337 (2006). https://doi. org/10.1016/.jmolcel.2006.01.011

37. F. Nakamura, M. Song, J.H. Hartwig, T.P. Stossel, Documentation and localization of force-mediated Filamin A domain perturbations in moving cells. Nat. Commun. 5, 1 (2014)

38. P. Kanchanawong, G. Shtengel, A.M. Pasapera, E.B. Ramko, M.W. Davidson, H.F. Hess, C.M. Waterman, Nanoscale architecture of integrin-based cell adhesions. Nature 468, 580 (2010). https://doi.org/10.1038/nature09621

39. M.D. Schaller, Paxillin: A focal adhesion-associated adaptor protein. Oncogene 20, 6459 (2001). https://doi.org/10.1038/sj.onc.1204786

40. G. Castoria, L. D'Amato, A. Ciociola, P. Giovannelli, T. Giraldi, L. Sepe, G. Paolella, M.V. Barone, A. Migliaccio, F. Auricchio, Androgen-induced cell migration: role of androgen receptor/filamin A association. PLoS ONE 6, 17218 (2011)

41. C.E. Turner, J.R. Glenney Jr., K. Burridge, Paxillin: A new vinculin-binding protein present in focal adhesions. J. Cell Biol. 111, 1059 (1990)

42. C.E. Turner, Paxillin and focal adhesion signalling. Nat. Cell Biol. 2, E231 (2000). https://doi.org/10.1038/35046659

43. C.D. Lynch, N.C. Gauthier, N. Biais, A.M. Lazar, P. Roca-Cusachs, C.-H.Yu, M.P. Sheetz, Filamin depletion blocks endoplasmic spreading and destabilizes force-bearing adhesions. Mol. Biol. Cell 22, 1263 (2011). https://doi.org/10. 1091/mbc.E10-08-0661

44. K.A. Beningo, M. Dembo, I. Kaverina, J.V. Small, Y.L. Wang, Nascent focal adhesions are responsible for the generation of strong propulsive forces in migrating fibroblasts. J. Cell Biol. 153, 881 (2001). https://doi.org/10.1083/jcb. 153.4.881

45. O. Krylyshkina, I. Kaverina, W. Kranewitter, W. Steffen, M.C. Alonso, R.A. Cross, J.V. Small, Modulation of substrate adhesion dynamics via microtubule targeting requires kinesin-1. J. Cell Biol. 156, 349 (2002). https://doi.org/10.1083/jcb. 200105051

46. O. Krylyshkina, K.I. Anderson, I. Kaverina, I. Upmann, D.J. Manstein, J.V. Small, D.K. Toomre, Nanometer targeting of microtubules to focal adhesions. J. Cell Biol. 161, 853 (2003). https://doi.org/10.1083/jcb.200301102

47. Y.M. Han, S.K. Lee, D.G. Jeong, S.E. Ryu, D.C. Han, D.K. Kim, B.M. Kwon, Emodin inhibits migration and invasion of DLD-1 (PRL-3) cells via inhibition of PRL-3 phosphatase activity. Bioorg. Med. Chem. Lett. 22, 323 (2012). https://doi. org/10.1016/j.bmcl.2011.11.008

48. J.H. Hwang, C.A. Smith, B. Salhia, J.T. Rutka, H.H. Jeong, C.A. Smith, B. Salhia, J.T. Rutka, The role of fascin in the migration and invasiveness of malignant glioma cells. Neoplasia 10, 149 (2008). https://doi.org/10.1593/neo.07909

\section{Publisher's Note}

Springer Nature remains neutral with regard to jurisdictional claims in published maps and institutional affiliations. 